\title{
Wavelength Assignment Considering Four Wave Mixing Using Genetic Algorithm
}

\author{
Helder A. Pereira, Daniel A. R. Chaves, Carmelo J. A. Bastos-Filho and Joaquim F. Martins-Filho
}

\begin{abstract}
This article presents a new strategy to assign wavelengths in an optical link considering the four wave mixing effect in the optical fiber. The four wave mixing effect is taken into account by using a formulation that calculates the fiber noise figure for each wavelength. We analyze the assignments when there are additions and droppings of wavelengths, considering the best and satisfactory assignments using the exhaustive and genetic algorithms, respectively. We obtain the distributions of wavelengths from a genetic algorithm, which considers the quality of service criterion based on bit error rate. We show that our strategy is capable of determining assignments of wavelength, the maximum number of wavelengths and their positions in the grid to minimize the impact of the four wave mixing effect.
\end{abstract}

Index Terms-Four Wave Mixing, Genetic Algorithm, Optical Networks, Wavelength Assignment.

\section{INTRODUCTION}

D UE to the demand on telecommunication data traffic, the number of channels in wavelength division multiplexing (WDM) optical-transmission systems have grown considerably. However, the transmission of many optical channels using WDM may cause nonlinear effects, like four wave mixing (FWM) [1], mainly in links using dispersion shifted (DSF) [2] or non-zero dispersion shifted fibers (NZDSF) [3]. As a result, estimating FWM efficiency is becoming very important, both for the design and evaluation of system performance. Hill et al [4] investigated the FWM process theoretically and experimentally in a single mode fiber at $514.5 \mathrm{~nm}$ wavelength, estimating the power generated by this nonlinear process. Shibata et al [5] used a highly sensitive technique that utilizes a heterodyne receiver and lock-in detection to evaluate FWM, and they proposed a formulation to include the efficiency dependence of the FWM with the channel frequency separation, fiber length, chromatic dispersion and dispersion slope. Song et al [2] reviewed all the formulations theoretically, and proposed more sophisticated models considering the depletion of the signals due to the fiber loss coefficient as well as the effect of self-phase modulation (SPM) and cross-phase modulation (XPM) on

Manuscript received June 17, 2006; revised November 12, 2008.

Helder A. Pereira is with Department of Electrical Engineering, University of Pernambuco, 52720-001, Recife-PE, Brazil. Email: helder.pereira@upe.poli.br.

Daniel A. R. Chaves and Joaquim F. Martins-Filho are with Photonics group, Department of Electronics and Systems, Federal University of Pernambuco, Rua Academico Helio Ramos, s/n, Bloco A, 4 Andar, Sala 408, Cidade Universitaria, 50740-530, Recife-PE, Brazil. Tel: (81) 2126-8210 r.257. Fax: (81) 2126-8215. Email: jfmf@ufpe.br.

Carmelo J. A. Bastos-Filho is with Department of Computing Systems, University of Pernambuco, 52720-001, Recife-PE, Brazil. Email: cjabf@dsc.upe.br
FWM efficiency. Whereas Inoue [6] considered the balance of the amplifier noise and the FWM noise in a cascaded link through a simple expression, determining the system input signal power range.

The noise figure (NF) [7] is an important parameter to determine the system performance and many formulations have been proposed. Summerfield and Tucker [8] and Obermann et al [9] evaluated the noise figure of a wavelength converter based on FWM in a semiconductor optical amplifier. In this case they consider the FWM power as the signal power, whereas the shot noise was used as a reference for the input noise power. The noise figure values obtained (around $20 \mathrm{~dB}$ ) are higher than the ones commonly reported for optical amplifiers (around $6 \mathrm{~dB}$ ). The noise figure parameter has also been employed in all optical networks for routing and wavelength assignment (RWA). Martins-Filho et al [10]-[12] proposed optical routing based on additive and multiplicative noise accumulation along optical transmission. Fonseca et al [13]-[15] proposed wavelength assignment algorithms to minimize the FWM effect on selected routes.

Bastos-Filho and Martins-Filho have recently proposed a new formulation to consider the FWM generated power and shot noise power as noise components in optical transmission systems [16]. Indeed, this formulation can quantify the signal degradation upon many additive noise sources interfering with the probe signal. They defined the fiber noise figure to include the FWM effect in the evaluation of transmission performance. The proposed model was validated by comparison to simulation results from a comercial software [17].

In this paper we propose a wavelength assignment algorithm using genetic algorithm that minimizes the impact of FWM effect on optical transmission, finding wavelength dispositions in the transmission grid that provide a required quality of service (QoS) in terms of bit error rate (BER).

This paper is organized as follows: In Section II, we review the formulation first proposed in [16] considering the FWM effect. In Section III, we describe the impact of FWM in terms of wavelength to assign. In Section IV and in Section V, we present our proposed genetic algorithm and we show the simulation results in terms of the established QoS criterion. In Section VI, we present the conclusions.

\section{NOISE FIGURE FORMULATION TO EVALUATE THE FOUR WAVE MIXING EFFECT}

In [18], Baney et al reported a noise figure definition in terms of measurable parameters. The noise factor $(F)$ is defined as 


$$
F=\frac{S N R_{\text {in }}}{S N R_{\text {out }}},
$$

where $S N R_{\text {in }}$ is the signal-to-noise ratio in the system input and $S N R_{\text {out }}$ is the signal-to-noise ratio in the system output. The noise figure is the noise factor expressed in decibel units. From the optoelectronic model [18], the $S N R$ is expressed as follows

$$
S N R=\frac{\left\langle i_{\text {Signal }}\right\rangle^{2}}{\left\langle\Delta^{2} i_{\text {Noise }}\right\rangle},
$$

where the term $\left\langle i_{\text {Signal }}\right\rangle^{2}$ is related to the photocurrent generated by the optical signal in a photodetector, and it is proportional to the detected electrical signal power, and $\left\langle\Delta^{2} i_{\text {Noise }}\right\rangle$ is the mean-square value of a single-sided noise power spectrum [18].

The formulation based on measurable parameters consists of an optoelectronic model and considers the scheme proposed in Fig. 1. In Fig. 1(a) there is no device between the source and the photodetector. One can evaluate an electrical signal power $\left(\left\langle i_{\text {Signal,in }}\right\rangle^{2}\right)$ and an electrical noise power $\left(\left\langle\Delta^{2} i_{\text {Noise,in }}\right\rangle\right)$ from the photodetector output. Then, in Fig. 1(b), one can insert an optical device between the source and the photodetector changing the electrical signal power $\left(\left\langle i_{\text {Signal,out }}\right\rangle^{2}\right)$ and the electrical noise power $\left(\left\langle\Delta^{2} i_{\text {Noise,out }}\right\rangle\right)$ in the photodetector output. In our case, the optical device is the transmission optical fiber.

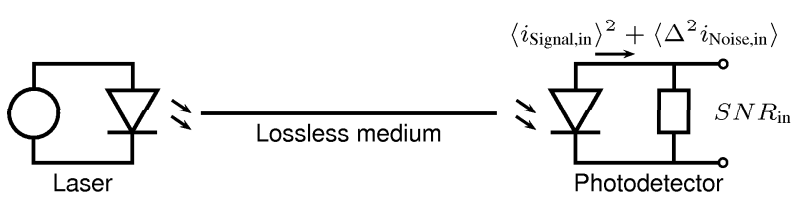

(a)

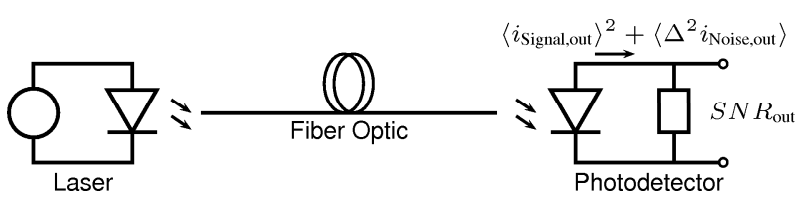

(b)

Fig. 1. Optoelectronic model that considers (a) no device, and (b) the optical fiber as a device between the source and the photodetector.

The photocurrent generated by the optical signal is

$$
\left\langle i_{\text {Signal }}\right\rangle=\mathcal{R} P_{\text {Signal }},
$$

where $P_{\text {Signal }}$ is the input optical power, $\mathcal{R}$ is the photodetector responsivity. The electrical noise power corresponds to

$$
\left\langle\Delta^{2} i_{\text {Noise }}\right\rangle=\mathcal{R}^{2} \int_{B_{\mathrm{e}}} S_{\text {Noise }}(f) d f,
$$

where $B_{\mathrm{e}}$ is the filter bandwidth positioned before the photodetector and $S_{\text {Noise }}(f)$ is the noise spectral power density.

Therefore, considering the shot noise as input reference noise (from Fig. 1(a)) we have [18]

$$
S N R_{\text {in }}=\frac{\left\langle i_{\text {Signal,in }}\right\rangle^{2}}{\left\langle\Delta^{2} i_{\text {Noise,Shot }}\right\rangle},
$$

where $\left\langle\Delta^{2} i_{\text {Noise,Shot }}\right\rangle$ quantifies the shot noise power component from the source. The shot-noise-limited input reference is widely used in the literature [18], it has been implemented by the noise figure instrument makers, and it is incorporated into the international definition of noise figure by the IEC (IEC 61291-1). Since shot noise arises from the particulate nature of uncorrelated photons or electrons, it represents the minimum practical noise obtainable in photonic or electronic circuits [18]. According to [18], (5) is equal to

$$
S N R_{\text {in }}=\frac{\mathcal{R}^{2} P_{\text {Signal }}^{2}}{2 q B_{\mathrm{e}} \mathcal{R} P_{\text {Signal }}} .
$$

For the fiber output, with the FWM generated power components plus shot noise, we have

$$
S N R_{\text {out }}=\frac{\mathcal{R}^{2}\left(P_{\text {Signal }} e^{-\alpha L}\right)^{2}}{2 q B_{\mathrm{e}} \mathcal{R} P_{\text {Signal }} e^{-\alpha L}+\left\langle\Delta^{2} i_{\text {FWM }}\right\rangle},
$$

where $\left\langle\Delta^{2} i_{\text {FWM }}\right\rangle$ corresponds to the noise square mean value due to additive noise components generated by FWM process, $L$ is the fiber length and $\alpha$ is the linear attenuation coefficient.

Therefore, from (6) and (7) the noise factor of the optical fiber can be written as

$$
F=\frac{\frac{\mathcal{R}^{2} P_{\text {Signal }}^{2}}{2 q B_{\mathrm{e}} \mathcal{R} P_{\text {Signal }}}}{\frac{\mathcal{R}^{2}\left(P_{\text {Signal }} e^{-\alpha L}\right)^{2}}{2 q B_{\mathrm{e}} \mathcal{R} P_{\text {Signal }} e^{-\alpha L}+\left\langle\Delta^{2} i_{\text {FWM }}\right\rangle}},
$$

that is equal to

$$
F=e^{\alpha L}\left(1+\frac{\left\langle\Delta^{2} i_{\mathrm{FWM}}\right\rangle}{2 q B_{\mathrm{e}} \mathcal{R} P_{\text {Signal }}} e^{\alpha L}\right) .
$$

We can analyze the wavelength assignments in terms of QoS criterion evaluating the $S N R_{\text {out }}$ from each wavelength in disposition using (9). We assume that the $S N R_{\mathrm{QoS}}$ which corresponds to $10^{-12}$ in terms of BER is approximately $22.9652 \mathrm{~dB}$ [19]. Since we are dealing with an analytical model we used 4 decimals for high accuracy.

Note that in the absence of FWM the noise factor, $F$ in (9), is equal to the reciprocal of the fiber loss. This is a consequence of the noise figure definitions used here [18], which considers the shot noise as the input reference noise. As shown in (7), the output electrical signal power is proportional to the square of the fiber loss, whereas the output electrical noise power (shot noise) is proportional to the fiber loss only. Therefore, the reciprocal of the fiber loss results from them. If one consider an arbitrary excess noise (higher than the shot noise) as the reference noise, the noise factor in the absence of FWM would be equal to 1 , since the fiber loss proportion on signal power and noise power would be the same. However, the excess noise at the input can mask the FWM noise produced by the fiber, reducing its relative impact on the noise factor. A consequence of this is a reduction in the accuracy of noise figure measurements that use excess noise at the input [18]. 


\section{A. Evaluation of additive noise components}

In this section we obtain the electrical noise power $\left(\left\langle\Delta^{2} i_{\text {Noise }}\right\rangle\right)$ when many additive noise components are interacting with the signal channel along the transmission. Considering a square law photodetector, the generated photocurrent obeys the following equation [7]

$$
i_{\mathrm{det}}=\mathcal{R} k\left|\sum_{i} \vec{E}_{i}\right|^{2},
$$

where $i_{\text {det }}$ is the detected photocurrent, $\vec{E}_{i}=E_{i} e^{j\left(\omega_{i} t+\phi_{i}\right)}$ is the $i$-th electromagnetic wave involved, $k$ is a constant equal to $\frac{n A \varepsilon_{0} c}{2}, n$ is the effective refractive index, $A$ is the effective area, $\varepsilon_{0}$ is the vacuum electrical permissivity and $c$ is the light speed.

Considering $\phi_{i}=0$, we can evaluate the square mean value of noise considering the beating process between the signal and the $n$ additive noise components. The result is given by $[20]$

$$
\begin{aligned}
\left\langle\Delta^{2} i_{\text {Noise }}\right\rangle & =\left\langle\Delta^{2} i_{\mathrm{FWM}}\right\rangle \\
& =\mathcal{R}^{2}\left[\left(\sum_{i=0}^{n} P_{i}+2 \sum_{i=0}^{n-1} \sum_{j=i+1}^{n} \sqrt{P_{i} P_{j}}\right)^{2}-P_{0}^{2}\right],
\end{aligned}
$$

where $P_{0}$ is the optical signal power, $P_{i}(i=1,2, \ldots, n)$ are the noise optical power components in the signal wavelength.

It is possible to evaluate $\left\langle\Delta^{2} i_{\mathrm{FWM}}\right\rangle$ using (11), with $P_{0}=P_{\text {Signal }} e^{-\alpha L}$, where $P_{0}$ is the signal power attenuated by the fiber loss, and considering $n$ additive noise power components generated by the FWM process in the signal wavelength. The FWM noise power can be obtained from the paper of Song et al [2], using equations (1-5) therein.

Using $\left\langle\Delta^{2} i_{\text {FWM }}\right\rangle$ and (9), one can evaluate the total fiber noise figure considering the FWM effect. This noise figure represents a figure of merit for the impact of the FWM effect on system performance, since it can be translated into degradation of the signal-to-noise ratio and BER due to FWM.

\section{WAVELENGTH ASSIGNMENT CONSIDERING THE IMPACT OF FWM EFFECT}

For a given grid size $\left(N_{\mathrm{t}}\right)$ and a number of wavelengths to assign $\left(N_{\mathrm{i}}\right)$, the optimization problem consists of finding the disposition of active wavelengths in the grid that minimizes the FWM effect. One can do that by testing every possible combination of active wavelengths in the grid (exhaustive algorithm). To represent a disposition of wavelengths (individual) in the grid we use a binary code, as shown in Fig. 2.

The arrows indicate an active wavelength in the grid, which are represented as bit 1 . Inactive wavelengths in the grid are represented as bit 0 . Every active wavelength has its SNR. Using (9), we can evaluate the degradation of SNR in each one considering the FWM effect and fiber attenuation.

We define the strength of the individual as the lower output SNR among all active wavelengths $\left(S N R_{<}\right)$. An individual

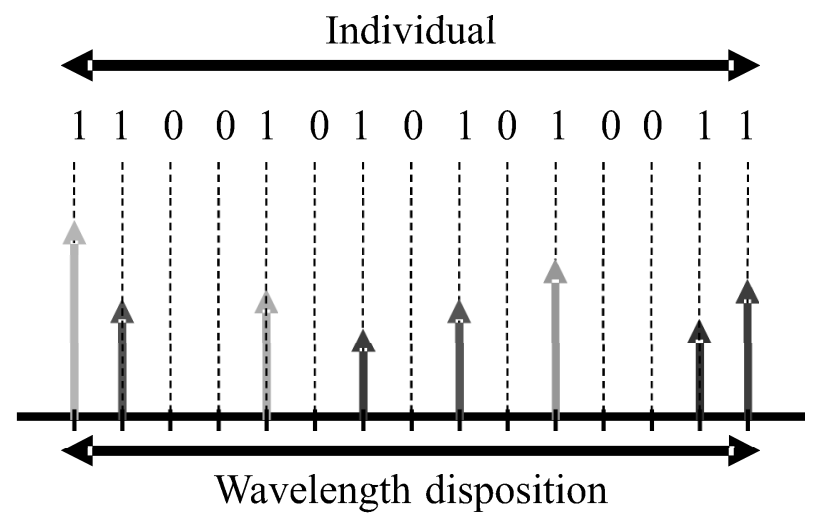

Fig. 2. Coded wavelength disposition considering $N_{\mathrm{t}}=15$ and $N_{\mathrm{i}}=8$.

that has higher $S N R_{<}$than other is considered as the stronger one. The process of calculating the $S N R_{\text {out }}$ from all active wavelengths in disposition and determining the $S N R_{<}$is called fitness evaluation. The output SNR should be higher than $22.9652 \mathrm{~dB}$ to satisfy the quality of service criterion, since it represents a bit error rate of $10^{-12}$.

In Fig. 3 we can determine the $S N R_{<}$from the best wavelength disposition using the exhaustive algorithm considering a variation of input optical power per channel, different frequency spacings, $N_{\mathrm{t}}=16, N_{\mathrm{i}}=8$ and the simulation parameters shown in Table I. Fig. 3 shows the maximum input optical power per channel for each frequency spacing. For example, considering $\Delta f=25 \mathrm{GHz}$, we have that $P_{\text {Signal }} \approx-12.6 \mathrm{dBm}$. Above this optical power the wavelength disposition does not satisfy the QoS criterion. For $\Delta f=50 \mathrm{GHz}$, we have $P_{\text {Signal }} \approx-9.3 \mathrm{dBm}$ and for $\Delta f=100 \mathrm{GHz}$, we have $P_{\text {Signal }} \approx 5.7 \mathrm{dBm}$.

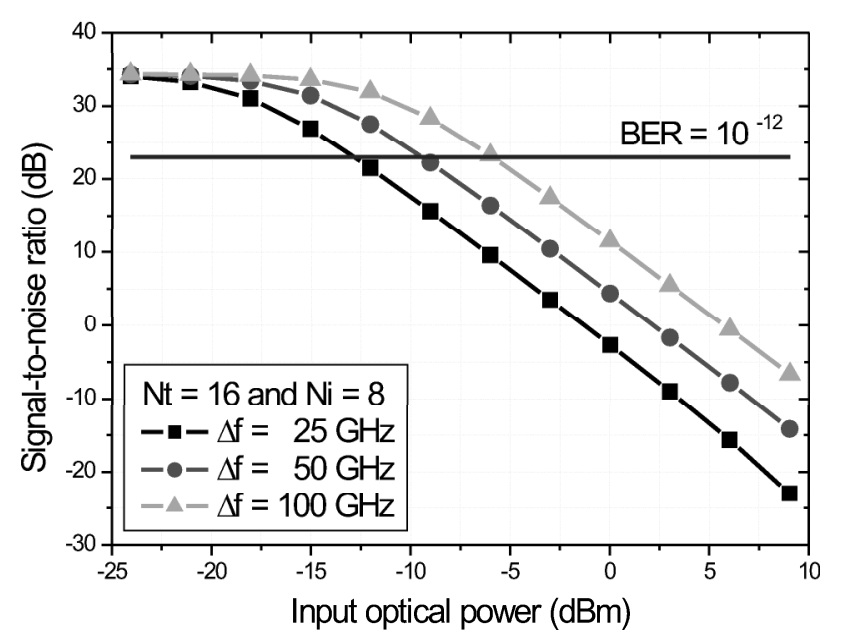

Fig. 3. Lower wavelength signal-to-noise ratio from the best wavelength disposition obtained from the exhaustive algorithm as a function of optical power per channel considering different frequency spacings.

In optical networks, considering dynamic traffic, wavelengths are added and dropped for establishing and finishing connection requests depending on the capacity of transmission and WDM equipments.

From the best wavelength disposition found by exhaustive algorithm considering $N_{\mathrm{t}}=16$ and $N_{\mathrm{i}}=7$, we can analyse 
TABLE I

SIMULATION PARAMETERS.

\begin{tabular}{c||c||c}
\hline Parameter & Value & Definition \\
\hline \hline$\lambda_{i}$ & $1550.12 \mathrm{~nm}$ & First wavelength of the grid. \\
$\lambda_{0}$ & $1544 \mathrm{~nm}$ & Zero dispersion wavelength. \\
$\alpha$ & $0.2 \mathrm{~dB} / \mathrm{km}$ & Optical fiber loss coefficient. \\
$L$ & $19.821 \mathrm{~km}$ & Link length. \\
$\Delta f$ & $50 \mathrm{GHz}$ & Frequency spacing. \\
$S N R_{\text {in }}$ & $38.5 \mathrm{~dB}$ & Input signal-to-noise ratio. \\
$S N R_{\mathrm{QoS}}$ & $22.9652 \mathrm{~dB}$ & Signal-to-noise ratio for QoS criterion. \\
$P_{\text {Signal }}$ & - & Input optical power per channel. \\
\hline
\end{tabular}

the $S N R_{<}$for each new disposition when a wavelength is added. Table II shows the different wavelength dispositions and their $S N R_{<}$values. We can note that the best disposition considering $N_{\mathrm{i}}=7$ has $S N R_{<} \approx 26.90 \mathrm{~dB}$. This value is above of $S N R_{\mathrm{QoS}}$ and represents a satisfactory disposition. By adding one wavelength to it we generate a new disposition with $N_{\mathrm{i}}=8$. For example, lighting $\lambda_{3}, \lambda_{4}, \lambda_{5}, \lambda_{7}, \lambda_{8}$, $\lambda_{10}, \lambda_{11}, \lambda_{12}$ or $\lambda_{14}$, we obtain new dispositions that does not satisfy the QoS criterion, becoming a non valid disposition in terms of BER.

TABLE II

ANALYSIS OF ONE WAVELENGTH ADDED FROM THE BEST DISPOSITION CONSIDERING $N_{\mathrm{I}}=7$.

\begin{tabular}{c||c||c}
\hline Wavelength disposition & Wavelength condition & $\mathbf{S} N R_{<}(\mathbf{d B})$ \\
\hline \hline 1100010010001011 & Best $\left(N_{\mathrm{i}}=7\right)$ & 26.90 \\
$11 \mathbf{1 0 0 1 0 0 1 0 0 0 1 0 1 1}$ & $\lambda_{3}$ on & 18.67 \\
1101010010001011 & $\lambda_{4}$ on & 22.38 \\
1100110010001011 & $\lambda_{5}$ on & 20.72 \\
1100011010001011 & $\lambda_{7}$ on & 20.52 \\
1100010110001011 & $\lambda_{8}$ on & 21.56 \\
1100010011001011 & $\lambda_{10}$ on & 21.33 \\
1100010010101011 & $\lambda_{11}$ on & 22.18 \\
1100010010011011 & $\lambda_{12}$ on & 21.36 \\
1100010010001111 & $\lambda_{14}$ on & 17.12 \\
0101001100100111 & Random $\left(N_{\mathrm{i}}=8\right)$ & 18.95 \\
1100101000101101 & Best $\left(N_{\mathrm{i}}=8\right)$ & 24.03 \\
\hline
\end{tabular}

In Table II, we can note that the best disposition considering $N_{\mathrm{i}}=8$ satisfies the QoS criterion, and it is different of the best considering $N_{\mathrm{i}}=7$. It means that adding wavelengths randomly from the best disposition, considering a specific $N_{\mathrm{i}}$ value, can affect the QoS of all wavelengths in disposition when FWM effect is taking into account. It's important to add wavelengths in some positions and change others in the disposition to minimize the FWM effect as we can note from the best dispositions considering $N_{\mathrm{i}}=7$ and $N_{\mathrm{i}}=8$.

Considering the best disposition of $N_{\mathrm{i}}=8$, we can turn off one wavelength and generate different dispositions to analyze the dropping of connections in an optical link. Table III shows the results of different dispositions generated from dropping one wavelength considering the best disposition for $N_{\mathrm{i}}=8$. All of them have $S N R_{<}$value above $S N R_{\mathrm{QoS}}$ and represent satisfactory dispositions. However, when we generated a disposition randomly, it did not satisfy the QoS criterion.
TABLE III

ANALYSIS OF ONE WAVELENGTH DROPPED FROM THE BEST DISPOSITION CONSIDERING $N_{\mathrm{I}}=8$.

\begin{tabular}{c||c||c}
\hline Wavelength disposition & Wavelength condition & $\mathbf{S} N R_{<}(\mathbf{d B})$ \\
\hline \hline 1100101000101101 & Best $\left(N_{\mathrm{i}}=8\right)$ & 24.03 \\
$\mathbf{0 1 0 0 1 0 1 0 0 0 1 0 1 1 0 1}$ & $\lambda_{1}$ off & 23.51 \\
1000101000101101 & $\lambda_{2}$ off & 23.81 \\
1100001000101101 & $\lambda_{5}$ off & 24.80 \\
1100100000101101 & $\lambda_{7}$ off & 24.45 \\
1100101000001101 & $\lambda_{11}$ off & 26.71 \\
1100101000100101 & $\lambda_{13}$ off & 25.94 \\
1100101000101001 & $\lambda_{14}$ off & 24.78 \\
1100101000101100 & $\lambda_{16}$ off & 25.09 \\
0100001100100111 & Random $\left(N_{\mathrm{i}}=7\right)$ & 19.26 \\
1100010010001011 & Best $\left(N_{\mathrm{i}}=7\right)$ & 26.90 \\
\hline
\end{tabular}

\section{GENETIC ALGORITHM}

Depending on $N_{\mathrm{t}}$ and $N_{\mathrm{i}}$, the use of the exhaustive algorithm to find the best solution can be time-consuming. Therefore, the use of a search algorithm to find a satisfactory solution is more advantageous. This is because for practical purposes one needs to find a wavelength disposition that satisfies a QoS criterion, rather than to find the best wavelength disposition among all possibilities. There are several search algorithms from computational intelligence, such as simulated annealing, tabu search, greedy search, genetic algorithms, differential evolution, particle swarm optimization, fish school search, ant colony optimization, harmony search, among others [21]. The three formers are not based on populations and have a higher chance to be trapped in local minima if the parameters are not well adjusted. The other options are more suitable for the sort of searching problem faced here. However, some of them were designed to solve problems in continuous search spaces [21]. Therefore, we believe that genetic algorithms are the best choice for discrete and finite search spaces, as with wavelengths distributions in optical links.

Genetic algorithm (GA) is a optimization process that is based on natural selection and genetic concepts idealized by Charles Darwin. These concepts consider that fitter individuals have more chances to survive in a specific environment [22].

The GA used to optimize the wavelength dispositions executes the following procedures:

- Initial population: With $N_{\mathrm{t}}$ and $N_{\mathrm{i}}$ defined, $P$ different individuals are determined by lighting $N_{\mathrm{i}}$ transmitters randomly in each individual.

- Crossover: Each individual belonging to population is allowed to cross once and is selected based on a probability value $\left(P_{\text {Cross }}\right)$. For a pair of individuals $\left(I_{1}\right.$ and $\left.I_{2}\right)$ selected, this operation chooses an active transmitter in $I_{1}$ and an inactive in $I_{2}$ randomly. Then, the states of transmitters are changed for both individuals. The same process is done considering an inactive transmitter in $I_{1}$ and other active in $I_{2}$. The new individuals are added to population only if they are different from the others belonging to the population.

- Mutation: An individual belonging to the population is selected based on a probability value $\left(P_{\text {Mut }}\right)$. An active transmitter and other inactive one are chosen randomly 
in wavelength disposition and changed between them.

- Selection: $P$ individuals are selected with the best values of $S N R_{<}$to compose a new population.

Fig. 4 shows the evolutionary process executed by genetic algorithm. Our algorithm generates a initial population, evaluates the fitness of each individual and does the selection. If the stopping criterion is not reached, the algorithm performs the crossover and mutation operations. The fitness evaluation and selection operation are performed again and the evolutionary process continues until the maximum number of generations is reached or the stopping criterion is satisfied.

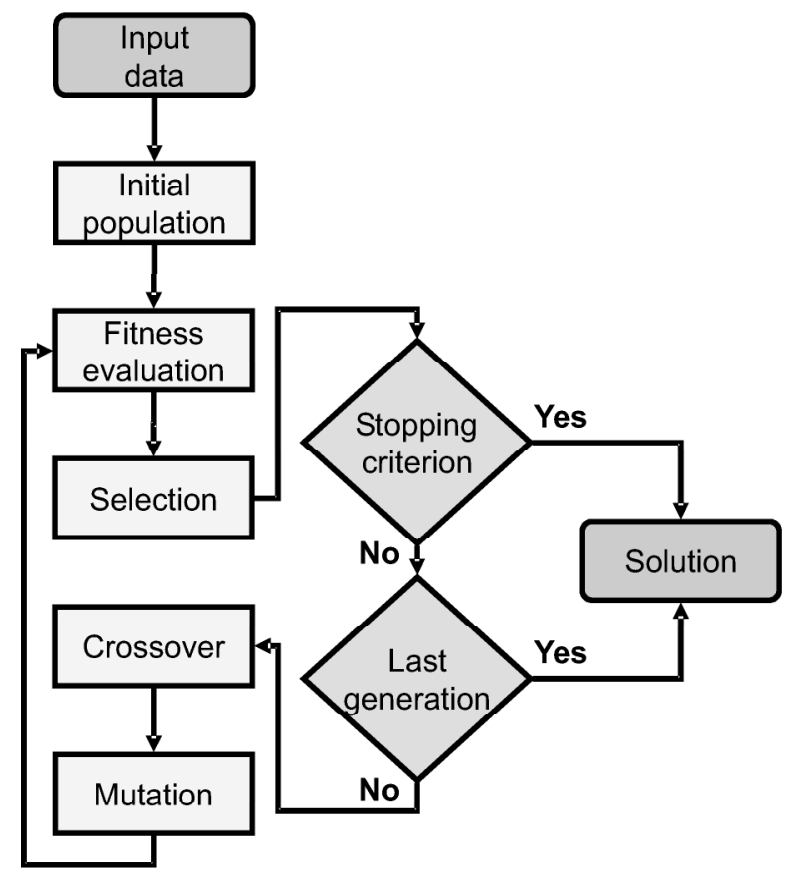

Fig. 4. Flow chart of the genetic algorithm used to optimize wavelength assignment in an optical link.

$P$ fitness evaluations are done at initial population. $2 P P_{\text {Cross }}$ evaluations are done according to the procedures executed during crossover operation and $\left(2 P P_{\text {Cross }}+P\right) P_{\text {Mut }}$ at the mutation operation. As the crossover and mutation operations are executed several generations during the evolutionary process, we have the following expression for the number of executed fitness evaluations:

$$
C_{\mathrm{F}}=P\left\{1+G\left[2 P_{\text {Cross }}+P_{\text {Mut }}\left(1+2 P_{\text {Cross }}\right)\right]\right\} .
$$

\section{A. Optimization of genetic parameters}

The parameters $P$ (initial population) and $G$ (maximum number of generations) are key parameters of the genetic algorithms, and their values impact on the final result and on the algorithm effort to find the result. Finding the values for these parameters is usually not a trivial task as they depend on the problem to solve, in our case on the parameters $N_{\mathrm{t}}$ and $N_{\mathrm{i}}$. We performed a set of simulations with our genetic algorithm, with different configurations of $N_{\mathrm{t}}$ and $N_{\mathrm{i}}$, to obtain some rules of choice for the $P$ and $G$ parameters. For these simulations we set the stopping criterion of the genetic algorithm as the best wavelength disposition found by exhaustive algorithm. Therefore, our genetic algorithm will stop only when it finds the best configuration. The number of generations and the number of fitness evaluations $\left(C_{\mathrm{F}}\right)$ needed to find it are registered. The simulation parameters are presented in Table I and Table IV.

TABLE IV

GENETIC PARAMETERS.

\begin{tabular}{c||c||c}
\hline Parameter & Value & Definition \\
\hline \hline$P$ & - & Initial population. \\
$G$ & - & Number of generations. \\
$P_{\text {Cross }}$ & $50 \%$ & Crossover probability. \\
$P_{\text {Mut }}$ & $5 \%$ & Mutation probability. \\
\hline
\end{tabular}

Fig. 5(a) and Fig. 5(b) show the evolution of the average of these parameters as a function of $P$, for $N_{\mathrm{t}}$ of 20 and $N_{\mathrm{i}}$ of 10 , for signal power of $-10 \mathrm{dBm}$ per channel. Every point represents the average result of 100 simulations. Fig. 5(a) shows that the value of $P$ to minimize $C_{\mathrm{F}}$ is around 85 . Whereas Fig. 5(b) shows that for this value of $P, G$ is 37. However, both figures show that $C_{\mathrm{F}}$ and $G$ have little dependence on $P$, for $P$ larger than 60 . We performed similar simulations with the configurations $N_{\mathrm{t}} / N_{\mathrm{i}}$ as $12 / 6$ and $16 / 8$, and the results are summarized in Table V. From these simulations we concluded that if one assumes $P=100$ and $G=100$ for any configuration, the $C_{\mathrm{F}}$ will not be far from the minimum value.

Table $\mathrm{V}$ also shows the parameter $R_{\text {Exhaus-GA, which we }}$ define as the ratio of the number of fitness evaluations executed by the exhaustive algorithm, by the number of fitness evaluations executed by the genetic algorithm. The latter is given by (12), and the former is given by

$$
C_{\mathrm{F}}=\frac{N_{\mathrm{t}} !}{\left(N_{\mathrm{t}}-N_{\mathrm{i}}\right) ! N_{\mathrm{i}} !} .
$$

TABLE V

GENETIC PARAMETERS FOR MINIMUM VALUE OF FITNESS EVALUATION.

\begin{tabular}{c||c||c||c}
\hline Configuration & $\mathbf{P}$ & $\mathbf{G}$ & $\mathbf{R}_{\text {Exhaus-GA }}$ \\
\hline \hline$N_{\mathrm{t}}=12 / N_{\mathrm{i}}=6$ & 30 & 11 & 2.42 \\
$N_{\mathrm{t}}=16 / N_{\mathrm{i}}=8$ & 50 & 18 & 12.88 \\
$N_{\mathrm{t}}=20 / N_{\mathrm{i}}=10$ & 85 & 37 & 51.77 \\
\hline
\end{tabular}

This parameter shows that for the smaller configuration $\left(N_{\mathrm{t}}=12\right.$ and $N_{\mathrm{i}}=6$ ) the genetic algorithm does less than half the number of fitness evaluations of the exhaustive algorithm. And for larger configurations the advantage of using the genetic algorithm further increases, since it performs fewer calculations to obtain the same result, which also impacts on the overall response time.

Although the obtained values of $P=100$ and $G=100$ are good enough for most of the medium and large configurations, it may not be as good for small configurations. In such cases these large values may lead the genetic algorithm to perform more fitness evaluations than the exhaustive algorithm. To avoid this possibility we developed 


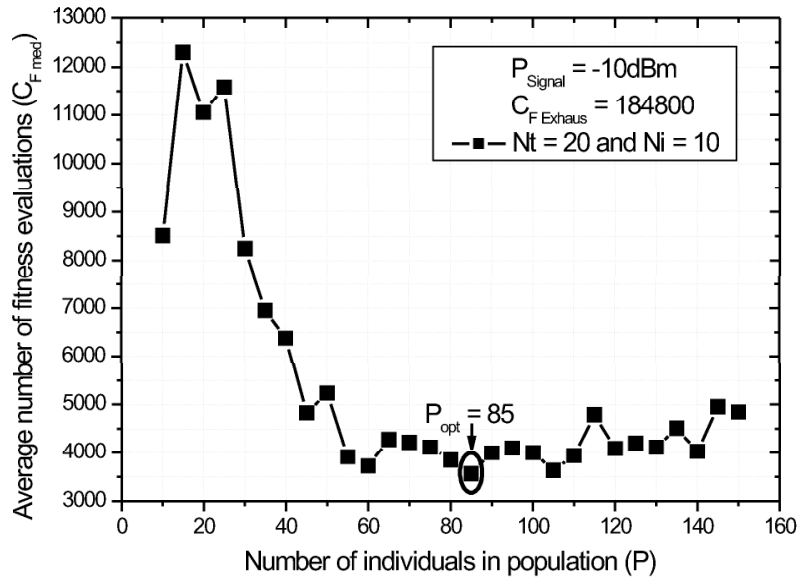

(a)

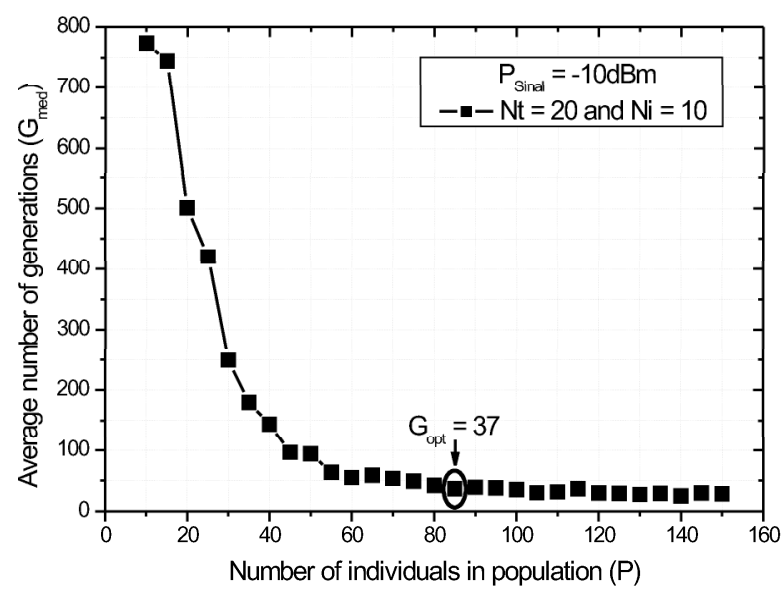

(b)

Fig. 5. Simulation results for (a) the average number of fitness evaluations and (b) average number of generations (b) as a function of $P$, for $N_{\mathrm{t}}=20$ and $N_{\mathrm{i}}=10$, and $P_{\text {Signal }}=-10 \mathrm{dBm}$.

the following procedures to obtain the initial values of $P$ and $G$, which is diagramed in Fig. 6:

- Input data: $N_{\mathrm{t}}, N_{\mathrm{i}}, P_{\text {Cross }}$ and $P_{\text {Mut }}$.

- Evaluate the number of fitness evaluations executed by exhaustive algorithm $\left(C_{\mathrm{F}}\right.$ Exhaus $)$ using (13).

- $P$ fitness evaluations are executed by genetic algorithm at initial population. Therefore, the initial parameter used by GA ( $P=100)$ can be compared with $C_{\mathrm{F} \text { Exhaus. }}$.

- If $C_{\mathrm{F} \text { Exhaus }}$ is less than $100, P$ assumes the $C_{\mathrm{F}}$ Exhaus value and $G$ is equal to zero.

- If $C_{\mathrm{F} \text { Exhaus }}$ is larger than $100, P$ is set to $100 . G$ is determined by the value between $G_{0}$ and 100 , where $G_{0}$ is the integer part of the following expression obtained from (12)

$$
\begin{aligned}
& G_{0}= \\
& \left.\qquad\left(\frac{C_{\mathrm{F} \text { Exhaus }}}{P}-1\right) \frac{1}{2 P_{\text {Cross }}+P_{\text {Mut }}\left(1+2 P_{\text {Cross }}\right)}\right\rfloor
\end{aligned}
$$

Therefore, $G_{0}$ is the maximum number of generations for the genetic algorithm to perform fewer fitness evaluations than the exhaustive algorithm ( $C_{\mathrm{F}}$ Exhaus).

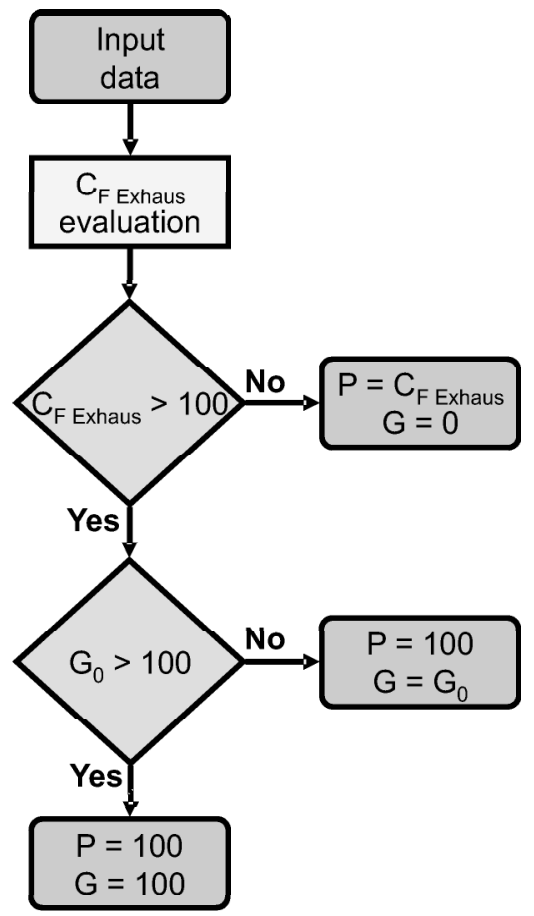

Fig. 6. Optimization steps to find $P$ and $G$ parameters for the genetic algorithm for wavelength assignment, for any $N_{\mathrm{t}}$ and $N_{\mathrm{i}}$.

These simple steps guarantee that the genetic algorithm will obtain the solution for any values of $N_{\mathrm{t}}$ and $N_{\mathrm{i}}$ performing fewer calculations than the exhaustive algorithm. The genetic parameters $P_{\text {Cross }}$ and $P_{\text {Mut }}$ used here and shown in Table IV are typical from any implementation of genetic algorithm [23]. Using these parameters along with the procedure described in this section will result in a better algorithm than the exhaustive search for any optical system. However, for some systems it may be more efficient than for others.

\section{GENETIC ALGORITHM FOR WAVELENGTH ASSIGNMENT CONSIDERING FWM FOR DIFFERENT CONFIGURATIONS}

For the purpose of optimization of wavelength assignment in an optical link considering the FWM effect, we set the stopping criterion of the genetic algorithm as the $S N R_{<}$equal to $22.9652 \mathrm{~dB}$, which guarantees a bit error rate of $10^{-12}$. Therefore, the solution obtained from the genetic algorithm is a satisfactory solution, rather than the best one.

Fig. 7 shows the average number of generations and fitness evaluations performed by the genetic algorithm to obtain a satisfactory solution for 3 different configurations of $N_{\mathrm{t}}$ and $N_{\mathrm{i}}$, as a function of the input optical power per channel. The number of fitness evaluations for the exhaustive algorithm is given for comparison. It can be seen that for low optical powers the genetic algorithm finds a satisfactory solution for the wavelength assignment in the initial population, with $G=0$, since in this case the FWM effect is negligible. As the optical power per channel increases, the FWM effect becomes important and the genetic algorithm needs to evolve for several generations to find a satisfactory solution for the wavelengths distribution. Fig. 7 also shows, for each configuration, the $S N R_{<}$of the last satisfactory solution, which occurs at $-8.54 \mathrm{dBm}$ for $12 / 6,-9.48 \mathrm{dBm}$ for $16 / 8$, and $-10.44 \mathrm{dBm}$ 
for 20/10. For higher optical powers the genetic algorithm does not find a satisfactory solution. However, in any case, the genetic algorithm does fewer fitness evaluations than the exhaustive algorithm.

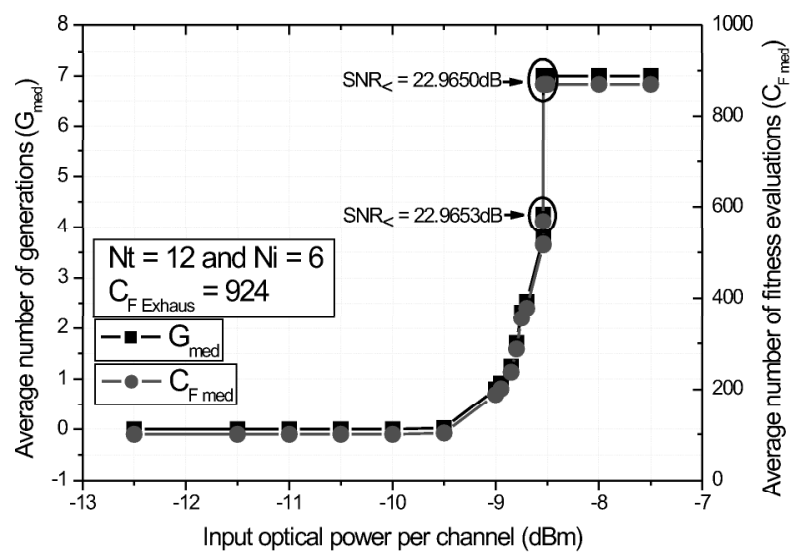

(a) $N_{\mathrm{t}}=12$ and $N_{\mathrm{i}}=6$.

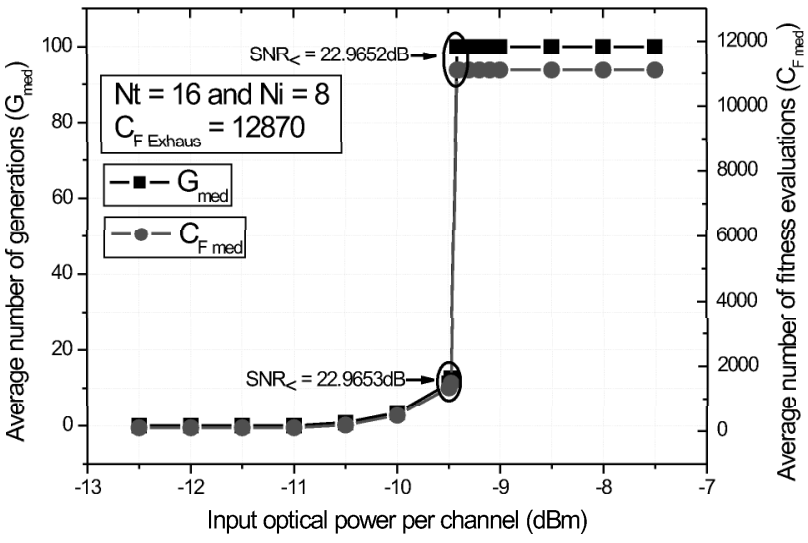

(b) $N_{\mathrm{t}}=16$ and $N_{\mathrm{i}}=8$.

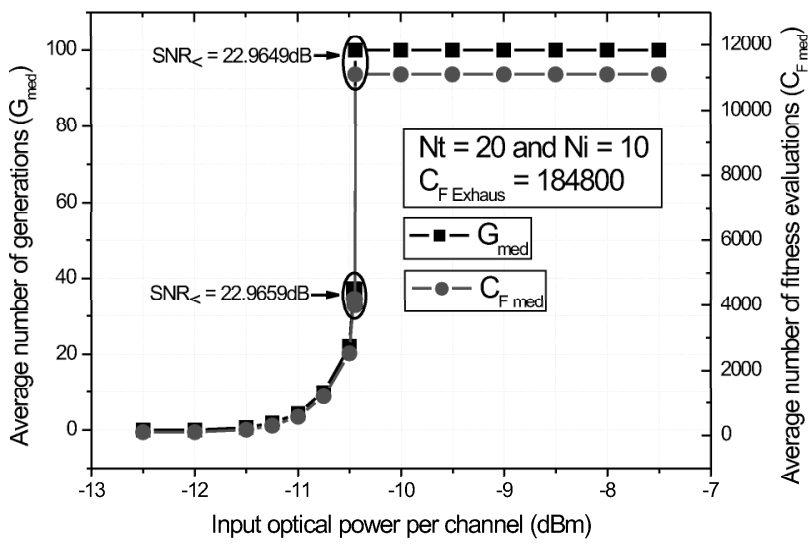

(c) $N_{\mathrm{t}}=20$ and $N_{\mathrm{i}}=10$.

Fig. 7. Average number of generations and fitness evaluations performed by the genetic algorithm as a function of the optical power per channel, for different configurations of $N_{\mathrm{t}}$ and $N_{\mathrm{i}}$.

Fig. 8 shows the $S N R_{<}$of the best solutions obtained from the exhaustive algorithm, as a function of the input optical power per channel, for different configurations of $N_{\mathrm{t}}$ and $N_{\mathrm{i}}$. As expected, the $S N R_{<}$decreases for higher input powers, since the FWM effect increases with the optical power. For optical powers below $-25 \mathrm{dBm}$ per channel the effect of FWM is negligible and the $S N R_{<}$is affected only by the fiber loss. And the maximum optical powers to obtain a $S N R_{<}$above the QoS limit is very similar to the ones obtained from the genetic algorithm given in Fig. 7. Therefore, Fig. 8 shows that our genetic algorithm is very efficient to find satisfactory solutions, even when these solutions are difficult to find, which is the case of high optical powers. This is because for high optical powers, there is a point where the best solution is the only satisfactory solution, which can be found by the genetic algorithm due to its evolution process, for many generations.

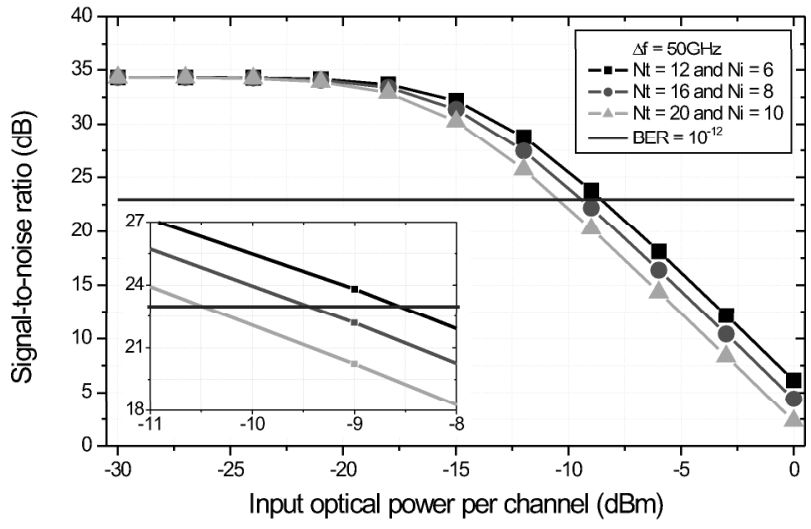

Fig. 8. $S N R_{<}$of the best wavelength disposition as a function of the optical power per channel, for different configurations of $N_{\mathrm{t}}$ and $N_{\mathrm{i}}$. The inset shows the details of the curves at the BER line crossing point.

\section{CONCLUSIONS}

We have reviewed the formulation first proposed by BastosFilho and Martins-Filho [16] that calculates the impact of FWM effect on the signal-to-noise ratio of the transmitted channels in a WDM optical link. Although the formulation considers single optical links, for multi-span systems with optical amplifiers one can use the cascade of noisy components to obtain the total noise factor. We proposed and demonstrated a genetic algorithm that uses this formulation to optimize the distribution of wavelengths to be used for wavelength assignment in optical links. This algorithm takes into account the grid size, the number of wavelengths to assign, the signal powers, fiber type, and frequency spacing between WDM signals. The proposed genetic algorithm finds solutions that satisfy a pre-determined quality of service criterion, which is the bit error rate bellow $10^{-12}$ for any transmitting channel. Moreover, we showed that our proposed genetic algorithm finds this solution executing fewer computer operations than an algorithm that tests all possible solutions. We believe that our proposed genetic algorithm has applications in off-line calculations for wavelength assignment in transparent optical networks.

\section{ACKNOWLEDGMENT}

The authors acknowledge the financial support from CAPES and $\mathrm{CNPq}$ (Brazilian Research Councils). 


\section{REFERENCES}

[1] N. Biryukov, O. Tytarchuk, and N. Triska, "Evaluation of four-wave mixing products in c-range," in Proceedings of the 4th International Conference on Transparent Optical Networks, vol. 1. IEEE, April 2002, pp. 229-232.

[2] S. Song, C. Allen, K. Demarest, and R. Hui, "Intensity-dependent phasematching effects on four-wave mixing in optical fibers," Journal of Lightwave Technology, vol. 17, no. 11, pp. 2285-2290, November 1999.

[3] S. Song, C. Allen, K. Demarest, L. Pelz, X. Fang, and Y. Pua, "Experimental study of four wave mixing in non-zero dispersion fiber," P. of Lasers and L. . t. A. M. Electro-Optics Society Annual Meeting, Eds., vol. 2, LEOS. IEEE, November 1997, pp. 224-225.

[4] K. O. Hill, D. Johnson, B. S. Kawasaki, and R. I. MacDonald, "Cw three-wave mixing in single mode fibers," Journal of Applied Physics, vol. 49, no. 10, pp. 5098-5106, October 1978.

[5] N. Shibata, R. Braun, and R. Waarts, "Phase-mismatch dependence of efficiency of wave generation through four-wave mixing in a single mode fiber," Journal of Quantum Electronics, vol. 23, no. 7, pp. 1205-1211, July 1987.

[6] K. Inoue, "A simple expression for optical fdm network scale considering fiber four-wave mixing and optical amplifier noise," Journal of Lightwave Technology, vol. 13, no. 5, pp. 856-861, May 1995.

[7] H. A. Haus, "Noise figure definition valid from rf to optical frequencies," Journal of Selected Topics in Quantum Electronics, vol. 6, no. 2, pp. 240-247, 2000.

[8] M. A. Summerfield and R. S. Tucker, "Noise figure and conversion efficiency of four-wave mixing in semiconductor optical amplifiers," Electronics Letters, vol. 31, no. 14, pp. 1159-1160, July 1995.

[9] K. Obermann, I. Koltchanov, K. Petermann, S. Diez, R. Ludwig, and H. Weber, "Noise analysis of frequency converters utilizing semiconductor-laser amplifiers," Journal of Quantum Electronics, vol. 33, no. 1, pp. 81-88, January 1997.

[10] J. F. Martins-Filho, C. J. A. Bastos-Filho, S. C. Oliveira, E. A. J. Arantes, E. Fontana, and F. D. Nunes, "Novel routing algorithm for optical networks based on noise figure and physical impairments," in Proceedings of ECOC, vol. 3. OSA, 2003, pp. 856-857.

[11] J. F. Martins-Filho, C. J. A. Bastos-Filho, E. A. J. Arantes, S. C. Oliveira, L. D. Coelho, J. P. G. de Oliveira, R. G. Dante, E. Fontana, and F. D. Nunes, "Novel routing algorithm for transparent optical networks based on noise figure and amplifier saturation," Proceedings of IMOC, vol. 2, pp. 919-923, September 2003.

[12] J. F. Martins-Filho, C. J. A. Bastos-Filho, E. A. J. Arantes, S. C. Oliveira, F. D. Nunes, R. G. Dante, and E. Fontana, "Impact of device characteristics on network performance from a physical-impairmentbased routing algorithm," Optical Fiber Communication Conference, vol. 1, pp. 23-27, February 2004.

[13] I. E. Fonseca, M. R. N. Ribeiro, R. C. Almeida Jr., and H. Waldman, "Preserving global optical qos in fwm impaired dynamic networks," Eletronics Letters, vol. 40, no. 3, pp. 191-192, February 2004.

[14] I. E. Fonseca, R. C. Almeida Jr., M. R. N. Ribeiro, and H. Waldman, "Algorithms for fwm-aware routing and wavelength assignment," in Proceedings of IMOC, vol. 2. IEEE-MTS/SBMO, September 2003, pp. 707-712.

[15] I. E. Fonseca, R. C. Almeida Jr., H. Waldman, and M. R. N. Ribeiro, "Meeting optical qos requirements with reduced complexity in dynamic wavelength assignment," in Proceedings of First International Conference on Broadband Networks. IEEE, October 2004, pp. 331334.

[16] C. J. A. Bastos-Filho and J. F. Martins-Filho, "Noise figure model for transmission performance evaluation considering four wave mixing and source spontaneous emission," in Proceedings of IMOC. IEEEMTS/SBMO, July 2005, pp. 87-91.

[17] Linksim version 3.3 user manual. Rsoft Inc, 2002.

[18] D. Baney, P. Gallion, and R. S. Tucker, "Theory and measurements techniques for the noise figure of optical amplifiers," Optical Fiber Technology, vol. 6, pp. 122-154, 2000.

[19] K. Thyagarajan and A. K. Ghatak, An introduction to fiber optics, 1 st ed. Cambridge - USA, 1998.

[20] H. A. Pereira, D. A. R. Chaves, C. J. A. Bastos-Filho, and J. F. MartinsFilho, "Optimization of wavelength assignment in an optical link considering four wave mixing using genetic algorithm," in Proceedings of International Telecommunications Symposium, vol. 1. IEEE, September 2006, pp. 501-506.

[21] R. C. Eberhart and Y. Shi, Computational Intelligence: Concepts to Implementations, 1st ed. Morgan Kaufmann.
[22] K. F. Man, K. S. Tang, and S. Kwong, "Genetic algorithms: Concepts and applications," IEEE Transactions on Industrial Eletronics, vol. 43, no. 5, pp. 519-534, Outubro 1996.

[23] D. Bisbal, I. Miguel, F. Gonzlez, J. Blas, J. C. Aguado, P. Fernndez, J. Durn, R. Durn, R. M. Lorenzo, E. J. Abril, and M. Lpez, "Dynamic routing and wavelength assignment in optical networks by means of genetic algorithms," Photonic Network Communications, vol. 7, no. 1, pp. 43-58, January 2004.

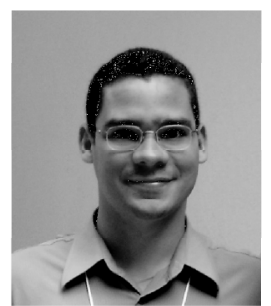

Helder A. Pereira was born in Paulista, Brazil, in 1980. He received the B.Sc. degree in Electronics Engineering from University of Pernambuco (UPE), Recife, Brazil, in 2000, the M.Sc. degree at Telecommunication National Institute (Inatel), Santa Rita do Sapucaí, Brazil, in 2002 and the PhD degree in Electrical Engineering at the Federal University of Pernambuco (IJFPF), Recife, Brazil, in 2007. He is a professor at the Electrical Engineering Department of University of Pernambuco (DEE-UPE).

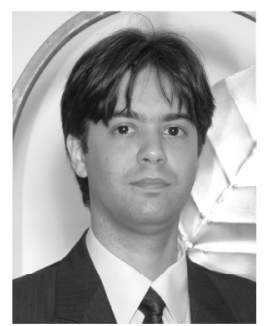

Daniel A. R. Chaves was born in Recife, Brazil, in 1981. He received the B.Sc. degree in Electronics Engineering from Federal University of Pernambuco (UFPE) in 2006. $\mathrm{He}$ is currently working toward the M.Sc. degree in Electrical Engineering at the Federal University of Pernambuco (UFPE).

$\mathrm{He}$ is currently working in laboratory of optical networks in the Laboratory of Optical Networks in the Photonics Group of UFPE. His interests are related to lightwave communication systems including optical amplifiers, high capacity WDM transmission, nonlinear effects, optical networking and computational intelligence applied to optical networks.

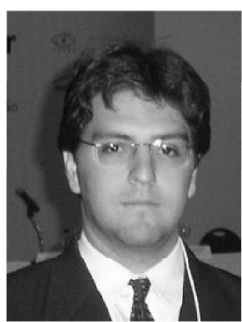

Carmelo J. A. Bastos-Filho was born in Recife, Brazil, in 1978. He received the B.Sc. degree in Electronics Engineering from Federal University of Pernambuco (UFPE) in 2000. He received the M.Sc. and $\mathrm{PhD}$ degrees in Electrical Engineering from Federal University of Pernambuco (UFPE), in 2003 and 2005 , respectively. His interests are related to lightwave communication systems including optical amplifiers, high capacity WDM transmission, nonlinear effects, optical networking, and artificial intelligence. He also works developing intelligent algorithms for searching and optimization problems. He is currently a professor at the Department of Computing and Systems of University of Pernambuco (DSC-UPE). He is the coordinator of the research division of the Polytechnical School of Pernambuco. 


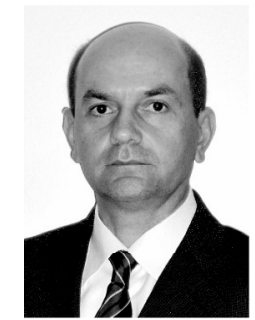

Joaquim F. Martins-Filho was born in Recife, Brazil, in 1966. He received the B.Sc. degree in Electronics Engineering from the Federal University of Pernambuco (UFPE), Recife, in 1989, and the M.Sc. degree in Physics from the same institution in 1991, studying nonlinear optics in optical fibers. He received his $\mathrm{PhD}$ degree in Electronics Engineering from the University of Glasgow, Scotland, in 1995, investigating ulfrafast optical pulse generation from mode-locked semiconductor quantum well lasers. Since 1998 he is an associate professor in the Photonics Group, Department of Electronics and Systems of UFPE, in Recife. His current interests are in devices, subsystems, transmission systems and networking for optical communications and optical sensors.

Prof. Joaquim Martins-Filho is a Member of OSA (Optical Society of America), SBMO (Brazilian Microwave and Optoelectronics Society), SBrT (Brazilian Telecommunication Society) and SBF (Brazilian Physics Society), as well as a Research Fellow of the National Research Council of Brazil $(\mathrm{CNPq})$. 